\title{
Resveratrol and lycium barbarum polysaccharide improve Qinling giant panda (Ailuropoda melanoleuca Qinlingensis) sperm quality during cryopreservation
}

\author{
Ruixue Zhang ${ }^{1,2+}$, Hemeng Dong ${ }^{1,2 \dagger}$, Pengpeng Zhao ${ }^{3 \dagger}$, Chunmei Shang ${ }^{1,2}$, Hang Qi ${ }^{1,2}$, Yongjie Ma ${ }^{1,2}$, \\ Chuxi Gao ${ }^{1,2}$, Danhui Zhang ${ }^{3}$, Jiena Shen ${ }^{3}$, Yinghu Lei ${ }^{3 *}$, Yaping Jin ${ }^{1,2^{*}}$ and Pengfei Lin ${ }^{1,2^{*}}$
}

\begin{abstract}
Background: Semen cryopreservation has become an essential tool for conservation efforts of the giant panda (Ailuropoda melanoleuca); however, it is severely detrimental to sperm quality. Evidence has shown that antioxidants have the potential to reverse cryopreservation-induced damage in sperm. The purpose of this study was to screen effective antioxidants that could retain sperm quality during cryopreservation and to determine the optimal dose. Seven antioxidant groups, including resveratrol (RSV $=50 \mu \mathrm{M}, \mathrm{RSV}=100 \mu \mathrm{M}, \mathrm{RSV}=150 \mu \mathrm{M}$ ), lycium barbarum polysaccharide ( $\mathrm{LBP}=2 \mathrm{mg} / \mathrm{mL}, \mathrm{LBP}=4 \mathrm{mg} / \mathrm{mL}$ ), laminaria japonica polysaccharides ( $\mathrm{LP}=1 \mathrm{mg} / \mathrm{mL}$ ) or combination $(\mathrm{LBP}=2 \mathrm{mg} / \mathrm{mL}, \mathrm{LJP}=1 \mathrm{mg} / \mathrm{mL}$ and $\mathrm{RSV}=100 \mu \mathrm{M})$ were assessed.

Results: RSV, LBP, LJP, or a combination of RSV, LBP, and LJP added to the freezing medium significantly improved sperm progressive motility, plasma membrane integrity, acrosome integrity, and mitochondrial activity during the cryopreservation process. Furthermore, the activities of glutathione peroxidase and superoxide dismutase were also improved. The levels of reactive oxygen species and malondialdehyde in semen were notably reduced. Hyaluronidase activity and acrosin activity were significantly increased in LBP-treated sperm. However, sperm total motility and DNA integrity were not significantly different between the groups.
\end{abstract}

Conclusions: RSV $(50 \mu \mathrm{M})$ or LBP $(2 \mathrm{mg} / \mathrm{mL})$ are the best candidate antioxidants for inclusion in the freezing medium to improve the quality of giant panda spermatozoa during semen cryopreservation.

Keywords: Qinling giant panda, cryopreservation, sperm quality, antioxidants, freezing medium

*Correspondence: 346779566@qq.com; yapingjin@163.com; linpengfei@nwsuaf.edu.cn

${ }^{\dagger}$ These authors contributed equally to this work and share first authorship.

${ }^{2}$ Key Laboratory of Animal Biotechnology, Ministry of Agriculture and Rural Affairs, Northwest A\&F University, Yangling 712100, Shaanxi, China

${ }^{3}$ Research Center for The Qinling Giant Panda, Rescue Base of Rare Wild Animals in Shaanxi Province, Louguantai 710402, China

Full list of author information is available at the end of the article

\section{Background}

Giant pandas (Ailuropoda melanoleuca) are one of the vulnerable animals in the world, and have survived on earth for at least eight million years [1, 2]. The Qinling giant panda (Ailuropoda melanoleuca qinlingensis), a subspecies of the giant panda, is a precious species because of its unique morphological characteristics and genetic features. From the fourth investigation report in 2015 by China's State Forestry Administration, approximately 345 individuals inhabited the Qinling 
Mountains, accounting for $18.51 \%$ of all wild giant pandas in China. Low mating opportunity is the main factor driving hypofertility in giant pandas, especially in captive-bred giant pandas, which have fewer chances of finding a suitable mate compared with wild giant pandas. Therefore, only $10 \%$ of the captive-bred giant pandas can mate naturally. Artificial insemination has become an effective biotechnology approach in conservation efforts of the giant panda; however, the efficiency of semen freezing is one of the major problems affecting the success of artificial insemination [3, 4]. Ultralow temperature cryopreservation may inflict damage to sperm cells; therefore, there is an increasing demand for improving semen cryopreservation methods for giant panda semen. This will be beneficial in maintaining the number of Qinling giant pandas, establishing a germplasm repository for the Qinling subspecies, and improving their conservation status.

Increasing evidence has shown that sperm cryopreservation induces the overproduction of reactive oxygen species (ROS), which are major deleterious factors affecting sperm quality [5]. Excessive ROS production leads to drastic changes in sperm membranes because natural antioxidants in the seminal plasma are diluted in the freezing medium before the cryopreservation process [6]. Recently, the addition of antioxidants to the sperm freezing medium for humans, horses, beers, dogs, goats, cattle, and boars has been demonstrated to significantly enhance sperm viability [7-11]. Multiple studies have confirmed that resveratrol (RSV), lycium barbarum polysaccharide (LBP), and laminaria japonica polysaccharides (LJP) can reduce excessive ROS production, thus limiting cryodamage [12-14]. RSV is a polyphenol found in plants such as grapes [15]. Recent studies have shown that RSV modulates lipid metabolism and has antiinflammatory, antioxidant, anti-allergic, and anti-cancer properties. Additionally, RSV displays protective effects against free radicals, cardiovascular diseases, and allergies [16]. Evidence from animal model studies on male fertilization have confirmed that RSV plays a promoting role in spermatogenesis by stimulating the hypothalamicpituitary gonadal axis [17]. In addition, RSV suppresses germ cell apoptosis, enhances penile erection, elevates steroidogenesis, and facilitates sperm motility and spermatogenesis in rats and mice [18]. LBP extracted from barbarums has been shown to improve the sperm quality. Moreover, the addition of LBP to the freezing medium not only increases the activity of antioxidant enzymes to inhibit apoptosis, but also enhances spermatogenesis in male mice with type 1 diabetes [19]. Multiple studies have indicated that LJP, a polysaccharide extracted from seaweed, has anti-coagulant, anti-arteriosclerosis, antitumor, and antiviral properties. In addition, studies have demonstrated that LJP can be added to the freezing medium, acting as a ROS scavenger to improve sperm quality during the freeze-thawing process [20].

In this study, we evaluated the potential effects of cryopreservation with RSV, LBP, or LJP on sperm motility, sperm plasma membrane integrity, acrosome integrity, mitochondrial activity, DNA integrity, antioxidant activities, and fertilization capacity. A further goal of this study was to provide effective antioxidants added in freezing medium to improve sperm resistance to cryopreservation.

\section{Results}

Antioxidant supplementation improves sperm motility of Qinling giant panda during cryopreservation

To explore the roles of antioxidants (RSV, LJP, and LBP) in giant panda sperm resistance to cryopreservation, we first assessed sperm total and progressive motility. We found no enhanced effect on the total motility of sperm exposed to RSV, LBP, or LJP alone at the indicated concentrations. Furthermore, the combination of RSV (100 $\mu \mathrm{M})$, LBP $(2 \mathrm{mg} / \mathrm{mL})$, and LJP $(1 \mathrm{mg} / \mathrm{mL})$ also did not change the total motility of freeze-thawing sperm when compared with the control (Fig. 1A, $P>0.05$ ). As shown in Fig. 1B, RSV alone had no effect on sperm progressive motility. Similarly, there was no significant difference between the LJP and control group, or the LBP $(4 \mathrm{mg} /$ $\mathrm{mL})$ group $(P>0.05)$. However, addition of LBP $(2 \mathrm{mg} /$ $\mathrm{mL})$ in freezing medium improved progressive motility compared with the control $(P<0.05)$. Furthermore, the combination of RSV $(100 \mu \mathrm{M})$, LBP $(2 \mathrm{mg} / \mathrm{mL})$, and LJP $(1 \mathrm{mg} / \mathrm{mL})$ notably improved sperm progressive motility after freeze-thawing process compared with the control $(P<0.05)$. Notably, a better post-thaw sperm progressive motility was observed in the combination group than in the LBP group $(2 \mathrm{mg} / \mathrm{mL})(P<0.05)$. These data indicated that supplementation with antioxidants did not change sperm total motility, but did improve sperm progressive motility.

\section{Antioxidant supplementation improves plasma membrane and acrosome integrity of Qinling giant panda sperm during cryopreservation}

Increasing evidence has shown that plasma membrane and acrosome integrity are associated with sperm motility [6]. Thus, we detected plasma membrane integrity and acrosome integrity in sperm treated with antioxidants. We found that supplementation with $50 \mu \mathrm{M}$ RSV and $100 \mu \mathrm{M}$ RSV markedly improved the plasma membrane integrity (Fig $2 \mathrm{~A}, P<0.05$ ), while there was no significant difference between the $50 \mu \mathrm{M}$ RSV and $100 \mu \mathrm{M}$ RSV treatment groups. Correspondingly, LJP (1 mg/mL) showed significant protective 


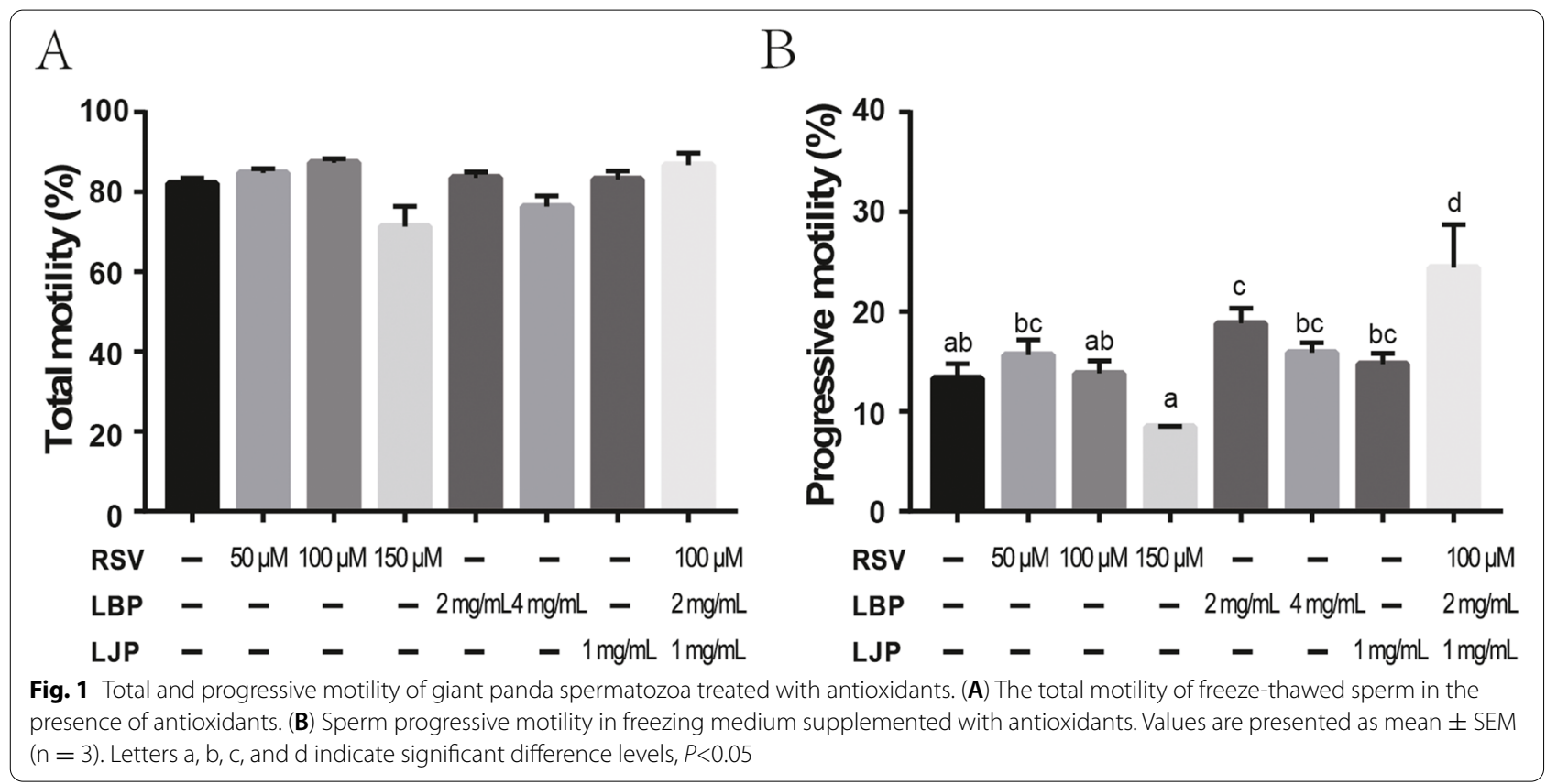

effects (Fig. 2A, $P<0.05)$. In addition, the combination of RSV $(100 \mu \mathrm{M})$, LBP $(2 \mathrm{mg} / \mathrm{mL})$, and LJP $(1 \mathrm{mg} / \mathrm{mL})$ protected the plasma membrane integrity compared with the control (Fig. 2A, $P<0.05$ ), yet showed no difference in the protection of plasma membrane integrity in comparison with RSV (50 / $100 \mu \mathrm{M})$ alone (Fig. 2A, $P$ $>0.05)$. However, LBP had no significant protective effect at all (Fig. 2A, $P>0.05$ ). These results suggested that RSV alone, LJP alone, and the combination had the ability to protect the plasma membrane integrity

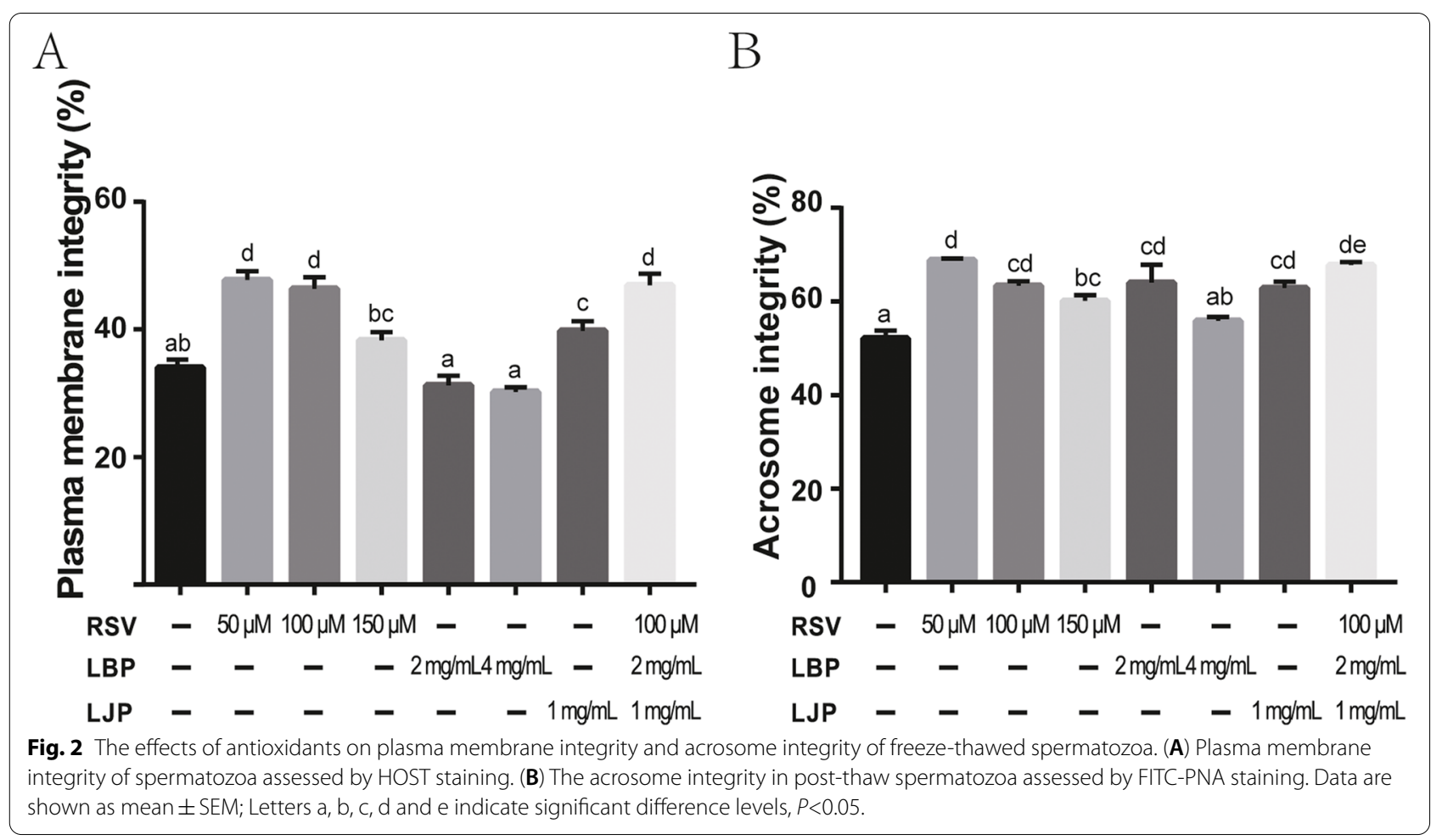


during cryopreservation, and $50 \mu \mathrm{M}$ of RSV was the optimal dose. As shown in Fig. 2B, we found that freezing medium with RSV alone improved acrosome integrity $(P<0.05)$. Furthermore, $1.0 \mathrm{mg} / \mathrm{mL} \mathrm{LJP}$ alone and $2.0 \mathrm{mg} / \mathrm{mL}$ LBP alone improved acrosome integrity $(P$ $<0.05)$. As expected, the combination of RSV $(100 \mu \mathrm{M})$, LBP $(2 \mathrm{mg} / \mathrm{mL})$, and LJP $(1 \mathrm{mg} / \mathrm{mL})$ increased acrosome integrity compared with the control $(P<0.05)$. Notably, RSV $(50 \mu \mathrm{M})$ and the combination were more effective than RSV $(100 \mu \mathrm{M})$, RSV $(150 \mu \mathrm{M})$, LBP $(2 \mathrm{mg} / \mathrm{mL})$, and LJP $(1 \mathrm{mg} / \mathrm{mL})$ alone. These results suggested that RSV, LJP, LBP, and their combinations at certain concentrations could protect acrosome integrity during cryopreservation.

\section{Antioxidant supplementation improves mitochondrial activity of Qinling giant panda sperm during cryopreservation}

To analyze the further protective effect of antioxidants, mitochondrial activity and DNA integrity were evaluated using PI/Rh123 staining and AO staining, respectively. The results showed that $50 \mu \mathrm{M}$ RSV and $100 \mu \mathrm{M}$ RSV significantly protected mitochondria from freeze-thawing injury (Fig. 3A, $P<0.05$ ), and mitochondrial activity was significantly increased in the freezing medium with addition of $2 \mathrm{mg} / \mathrm{mL} \mathrm{LBP}$ or $1 \mathrm{mg} / \mathrm{mL}$ LJP alone (Fig. 3A, $P$ $<0.05)$. Furthermore, the combination of RSV $(100 \mu \mathrm{M})$ and LBP $(2 \mathrm{mg} / \mathrm{mL})$ with LJP $(1 \mathrm{mg} / \mathrm{mL})$ significantly improved the mitochondrial activity of sperm when compared with the control (Fig. 3A, $P<0.05$ ). However, no significant effect on mitochondrial activity was observed in RSV $(150 \mu \mathrm{M})$ or LBP $(4 \mathrm{mg} / \mathrm{mL})$ groups. From the above results, $50 \mu \mathrm{M} \mathrm{RSV}, 1.0 \mathrm{mg} / \mathrm{mL} \mathrm{LJP}$, and the combined addition showed best protective effect in terms of mitochondrial activity (Fig. 3A, $P<0.05$ ). As shown in Fig. 3B, no significant effect on the DNA integrity of sperm was observed after treatment with RSV, LJP, LBP, or the combined addition compared with the control $(P>$ 0.05). Collectively, these data suggested that $50 \mu \mathrm{M}$ RSV and LJP $(1 \mathrm{mg} / \mathrm{mL})$ were the best candidates to improve the mitochondrial activity of sperm in freezing media.

\section{Antioxidant supplementation mitigates ROS levels and improves MDA, SOD, and GPX activity in cryopreserved sperm of Qinling giant panda}

To detect the protective capacity of antioxidants in thawed semen, antioxidant indices were determined using ELISA kits. Reactive oxygen species (ROS) levels were found to be significantly decreased in freezing media supplemented with $50 \mu \mathrm{M}$ RSV and $100 \mu \mathrm{M}$ RSV (Fig. 4A, $P<0.05$ ), but no significant change was found in the level of ROS after treatment with $150 \mu \mathrm{M}$ RSV, LBP, LJP, or their combination. In addition, resistance to ROS was observed in the $50 \mu \mathrm{M}$ and $100 \mu \mathrm{M}$ RSV groups, but better in the $100 \mu \mathrm{M}$ RSV group. As shown in Fig. 4B, all freezing media with RSV, LBP, LJP, or the combined addition had a significant protective effect against high levels of malondialdehyde (MDA) during freeze-thawing $(P<0.05)$. The effective protection of RSV $(50 \mu \mathrm{M})$ and RSV $(100 \mu \mathrm{M})$ was the highest (Fig. $4 \mathrm{~B}, P<0.05)$.

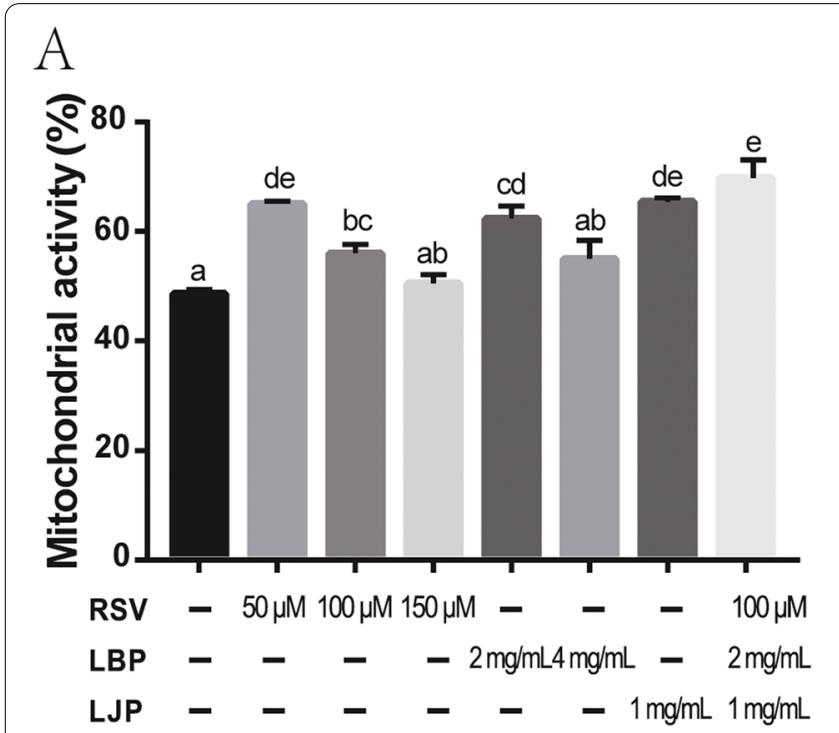

\section{B}

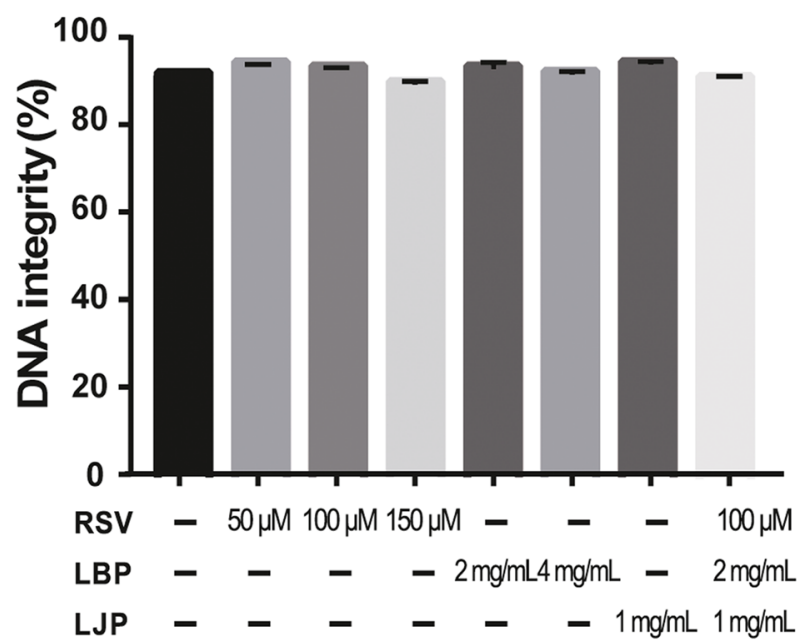

Fig. 3 Evaluation of the effect of antioxidants on mitochondrial activity and DNA integrity in giant panda spermatozoa. (A) Sperm mitochondrial activity assessed by staining with PI and Rh123. (B) DNA integrity evaluated using AO staining. Values are shown as mean $\pm \mathrm{SEM}(\mathrm{n}=3$ ). Letters a, $\mathrm{b}$, c, $d$ and e indicate significant difference levels, $P<0.05$. 


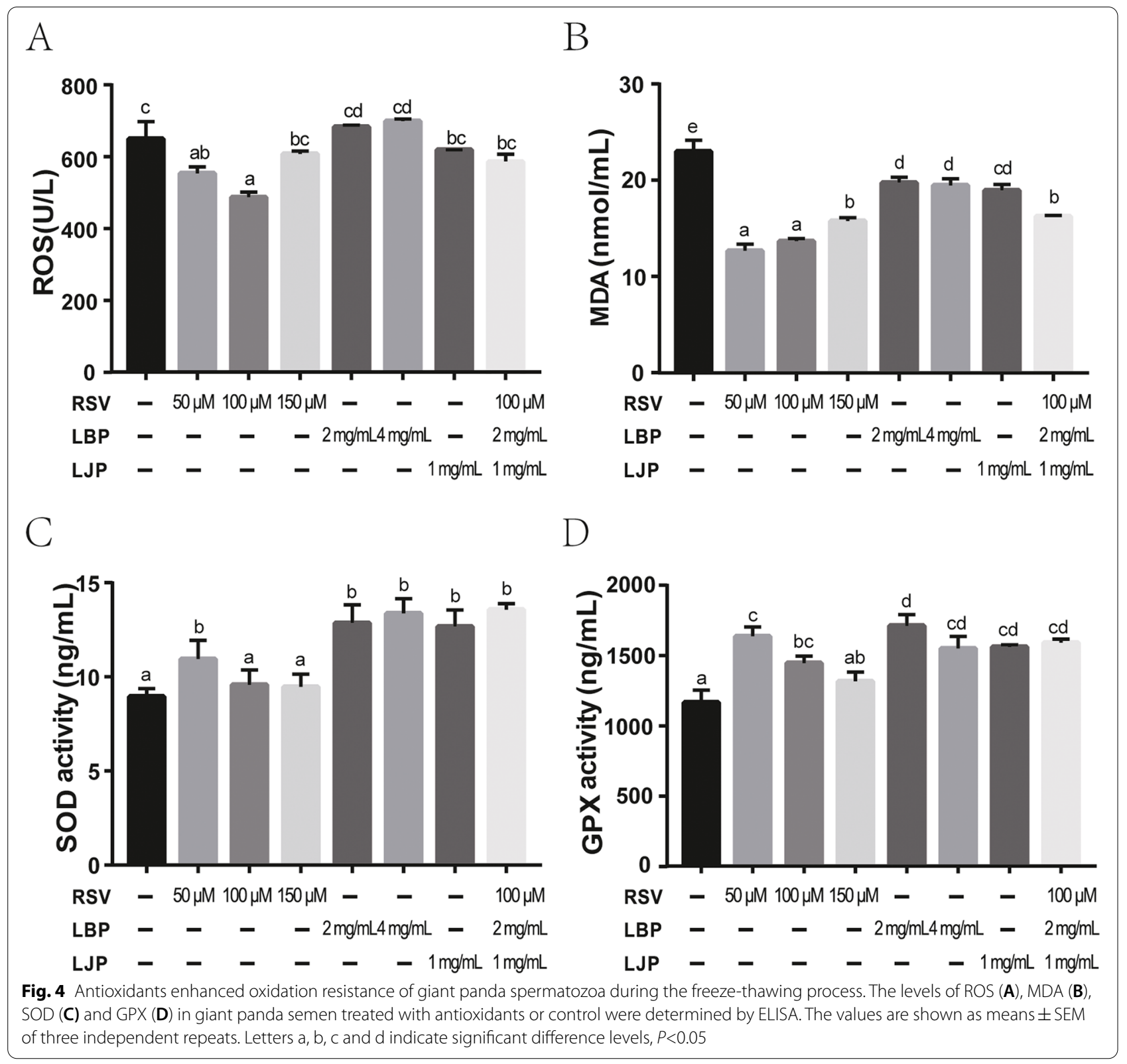

Addition of $50 \mu \mathrm{M}$ RSV, LBP alone, LJP alone and the combined addition contributed to increased superoxide dismutase (SOD) levels (Fig. $4 \mathrm{C}, P<0.05$ ). However, RSV $(100 \mu \mathrm{M}$ and $150 \mu \mathrm{M})$ did not have effect on SOD levels compared with the control (Fig. $4 \mathrm{C}, P>0.05$ ). Analysis of glutathione peroxidase (GPX) levels revealed that the facilitating effect of $50 \mu \mathrm{M}$ RSV alone and $100 \mu \mathrm{M}$ RSV alone was observed in sperm cryopreservation (Fig. 4D, $P$ $<0.05)$, but RSV $(150 \mu \mathrm{M})$ did not enhance GPX levels. LBP and LJP alone also improved the level of GPX in comparison with the control (Fig. 4D, $P<0.05$ ). Similarly, the combination of RSV $(100 \mu \mathrm{M})$, LBP $(2 \mathrm{mg} / \mathrm{mL})$, and
LJP (1 mg/mL) significantly increased the level of GPX compared to the control (Fig. 4D, $P<0.05$ ). Among the treatments, LBP $(2 \mathrm{mg} / \mathrm{mL})$ was the best candidate in elevating GPX levels. Collectively, these data indicated that antioxidants in the freezing medium decreased ROS and MDA levels while increased SOD and GPX levels.

\section{Supplementation of freezing media with antioxidants improves fertilization capacity of sperm of Qinling giant panda}

To analyze the fertilization ability of giant panda sperm after cryopreservation, protease assays were performed 

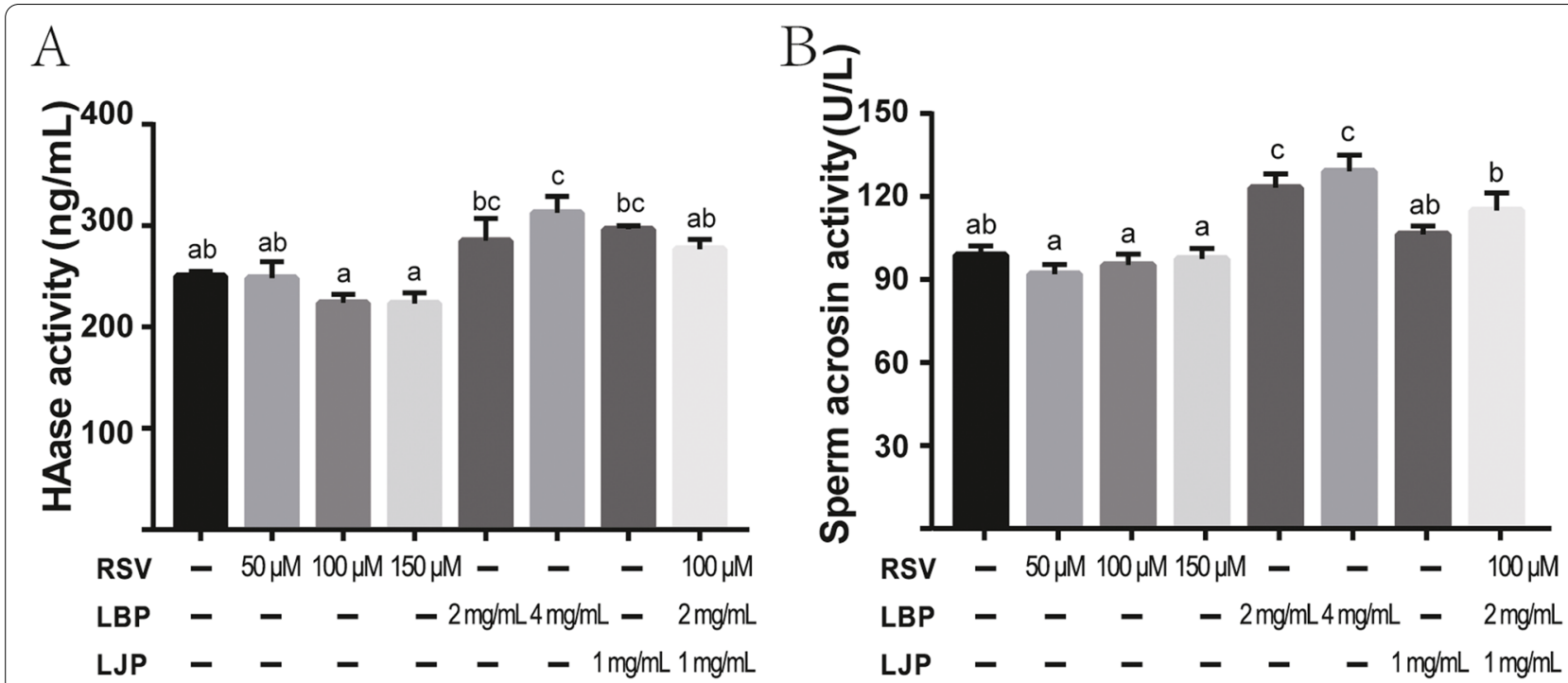

Fig. 5 Improvements in fertilizing capability in giant panda spermatozoa. (A) Sperm HAase content determined by ELISA. (B) Acrosin activity of spermatozoa measured by ELISA. All the experimental values are shown as the means \pm SEM of three repeats. Letters $a$, $b$ and $c$ indicate significant difference levels, $P<0.05$.

using ELISA. As shown in Fig. 5A, a significant difference in the hyaluronidase (HAase) content in sperm was found after treatment with LBP $(4 \mathrm{mg} / \mathrm{mL})$ compared with the control (Fig. 5A, $P<0.05$ ). However, no enhancement was observed in sperm treated with RSV, LBP $(2 \mathrm{mg} / \mathrm{mL}$ ), LJP, or their combination (Fig. $5 \mathrm{~A}, P>$ 0.05). As shown in Fig. 5B, supplementation of LBP with doses of $2 \mathrm{mg} / \mathrm{mL}$ and $4 \mathrm{mg} / \mathrm{mL}$ exerted a dramatic cryoprotective effect on sperm acrosomal protease (ACE) activity $(P<0.05)$, and the difference in sperm ACE activity between $2 \mathrm{mg} / \mathrm{mL}$ and $4 \mathrm{mg} / \mathrm{mL}$ was not significant (Fig. 5B, $P>0.05$ ). However, RSV, LJP, and the combined addition did not promote sperm ACE activity. Overall, these data indicated that the addition of LBP to the freezing medium improved the fertilization capacity.

\section{Discussion}

Giant pandas are one of the vulnerable species endemic to China, and adaptive for protection of the Qinling subspecies is the most pressing challenge. Apart from the establishment of nature reserves, semen cryopreservation of giant pandas is an effective approach to facilitate reproduction and sustain biodiversity. However, the negative effect of cryopreservation on the quality of giant panda sperm is inevitable. Substantial evidence has suggested that cryopreservation severely compromises plasma membrane fluidity, antioxidant ability, acrosome enzyme activity, mitochondrial potential, and motility, thereby affecting the sperm quality. Many researchers have investigated methods to protect the sperm from cryo-induced damage. Studies have further demonstrated that the addition of cryoprotective agents is important in reducing damage to sperm during cryopreservation. In cryoprotectant type, silicane-coated silica colloid particles were added to the freezing medium to improve the ability of spermatozoa fertilization of giant panda [21]. INRA96 freezing dilutions increased the quality of giant panda semen after thawing, compared to the TEST extender [22]. In addition, cryoprotectants, such as glycerol, sucrose, vitamin B12, vitamin E, vitamin C, dimethyl sulfoxide (DMSO), and trehalose were added to the freezing medium. At the transcript level, differentially expressed lncRNAs, miRNAs and mRNAs were analyzed in frozen and fresh sperm of giant pandas using high-throughput sequencing technology [23, 24]. The above evidence showed that the sperm of giant pandas suffered cryo-injury [25]. Previous reports have shown that RSV, LBP, and LJP added to semen improved the quality of sperm [7]. While the protective effect of antioxidants on ruminant sperm has been widely investigated, few studies have explored the optimum doses of LBP, LJP, and RSV for giant panda sperm cryopreservation. To develop new cryopreservation techniques and methods, novel findings regarding freezing medium of giant panda supplemented with antioxidants are in high demand. Because the cytotoxic effects rise with high concentrations, the optimal dose and concentration of LBP, LJP, and RSV were referenced from the dose in cattle, sheep, pigs, and other animals. In our study, seven groups of antioxidants were added to the freezing medium and positive effect on sperm quality was observed. This study provides an important 
reference for future improvements in cryopreservation of giant panda sperm.

Pioneering work previously showed that supplementation of buffalo semen freezing medium with RSV (50 $\mu \mathrm{M})$ could markedly improve the post-thaw quality of sperm [26]. Moreover, the addition of RSV $(10 \mu \mathrm{M}$ and $50 \mu \mathrm{M})$ to the freezing medium prior to cryopreservation also attenuated cryodamage and enhanced the motility of goat sperm [7]. Research has shown that 2 $\mathrm{mg} / \mathrm{mL} \mathrm{LBP}+1 \mathrm{mg} / \mathrm{mL}$ LJP could significantly improve the quality of Cashmere goat sperm [27]. In our study, we detected the protective effects of LBP, LJP, and RSV on giant panda sperm after cryopreservation. Similarly, we confirmed that supplementation of the freezing medium with $2 \mathrm{mg} / \mathrm{mL}$ LBP or a combination was effective at protecting giant panda sperm from cryoinjury. However, RSV and LJP alone had no protective effect on giant panda sperm motility. Interestingly, the dose of LBP applied to Cashmere goat spermatozoa can be considered to improve giant panda sperm motility. During the freeze-thaw process, the plasma membrane fluidity and permeability increased. The redistribution of antioxidant enzymes, phospholipids, and cholesterol is observed in the sperm plasma membrane and is responsible for structural and functional changes. In addition, plasma membrane integrity, which is involved in metabolism and osmotic pressure, is a critical factor in fertility [28]. Recently, evidence has confirmed that cryopreservation are detrimental to plasma membrane integrity and acrosome integrity in bulls, boars, rams, and sheep [26, 29-31]. To improve plasma membrane integrity and acrosome integrity, $50 \mu \mathrm{M}$ RSV was found to be an effective antioxidant for boar semen cryopreservation [13]. Supplementation of tris citric acid extender with $100 \mu \mathrm{M}$ RSV significantly enhanced the plasma membrane integrity of buffalo [32]. Furthermore, LJP and LBP exert protective effects on plasma membrane integrity and acrosome integrity [27]. Based on the antioxidant function of antioxidants, we further investigated whether antioxidants exerted a cryoprotective effect on giant panda sperm. Our study showed that 50 $\mu \mathrm{M}$ RSV, $100 \mu \mathrm{M}$ RSV, LJP alone and their combination had a cryoprotective effect on giant panda sperm plasma membrane integrity and acrosome integrity, which was consistent with the above-mentioned report. In addition, $50 \mu \mathrm{M}$ RSV and LBP $(2 \mathrm{mg} / \mathrm{mL})$ were beneficial to acrosome integrity. Recently, evidence from cryopreservation studies has confirmed that sperm with damaged DNA have been detected in cats, humans, and bovines [33-35]. Furthermore, antioxidants, extenders, and new approaches for humans have been used to improve sperm chromatin quality during freezing [33-35]. However, there were no protective effects of RSV, LBP, and
LJP on the DNA integrity of giant panda semen after cryopreservation in our study.

Mitochondrial ATP for energy homeostasis is generated by oxidative phosphorylation and ATP synthases, which plays a vital role in sperm motility [36]. Mitochondria are the primary organelles responsible for ROS production due to disturbances in the electron transport chain. In addition, the capacity of antioxidant enzymes against oxidative stress is significantly reduce because their abundance is diluted and thus have a negative effect on the cryopreservation results [37]. Therefore, excessive ROS levels can impair mitochondrial proteins, resulting in severely damaged mitochondrial activity [6, 38]. Recently, evidence has shown that the addition of RSV, LBP, and LJP to freeze-thawed semen could significantly improve the mitochondrial activity of sperm [7, 13, 27, 38]. In our study, we verified that the addition of $50 \mu \mathrm{M}$ RSV, $100 \mu \mathrm{M}$ RSV, $2 \mathrm{mg} /$ $\mathrm{mL}$ LBP, $1 \mathrm{mg} / \mathrm{mL} \mathrm{LJP}$ or the combined addition into the freezing medium improved mitochondrial activity of sperm, which was consistent with a previous study [13]. Previous reports have shown that cryopreservation is harmful to mitochondrial function and increases the production of ROS, and excessive production of ROS attacks polyunsaturated phospholipids and trigger elevation of MDA levels. In addition, the antioxidant activity of SOD and the level of GPX are reduced during cryopreservation. Finally, the semipermeable properties of the membrane are affected by peroxidative attack. However, the addition of RSV, LJP, and LBP to semen reduces the negative effects of excessive ROS levels [27, 38-40]. Consistent with a previous study, we further verified that $50 \mu \mathrm{M}$ RSV and $100 \mu \mathrm{M}$ RSV inhibited the production of ROS. In addition, our results showed that the addition of antioxidants prior to freezing protected sperm from cryodamage induced by MDA, whereas $50 \mu \mathrm{M}$ RSV was the best potential candidate as a cryoprotective agent. Recently, accumulating evidence has revealed that RSV, LJP and LBP could be beneficial by modulating activity of SOD and GPX [27, 41]. Our study verified that supplementation of the freezing medium with $50 \mu \mathrm{M}$ RSV , $2 \mathrm{mg} /$ $\mathrm{mL} \mathrm{LBP}, 1 \mathrm{mg} / \mathrm{mL} \mathrm{LJP}$, and combined addition significantly enhanced activity of SOD and GPX for oxygen radical $\left(\mathrm{O}_{2}^{-}\right)$scavenging. Therefore, supplementation of the freezing medium with antioxidants improved the enzymatic antioxidant activities of giant panda sperm.

In mammals, spermatozoa penetrate the zonal pellucida through the action of acrosomal enzymes. Among acrosomal enzymes, acrosin is identified as the most important factor, with a strong hydrolyzing activity. Evidence has shown that sperm mitochondrial activity affects human sperm acrosin activity [42]. In addition, 
HAase is important for digesting hyaluronic acid during the acrosome reaction process [43]. Therefore, HAase activity in sperm is responsible for fertilization. In our study, sperm ACE and HAase levels were improved in the $4 \mathrm{mg} / \mathrm{mL} \mathrm{LBP}$ treated group, and $2 \mathrm{mg} / \mathrm{ml} \mathrm{LBP}$ had protective effects on sperm ACE.

\section{Conclusion}

In summary, our study revealed that freezing medium supplemented with LJP, LBP, RSV, and their combination enhanced sperm motility, sperm plasma membrane integrity, acrosome integrity, and mitochondrial activity by increasing antioxidant enzyme levels and suppressing ROS production during the freezing process. $50 \mu \mathrm{M}$ RSV and $2.0 \mathrm{mg} / \mathrm{mL}$ LBP could be potential candidates as supplementation of the freezing medium against cryodamage to giant panda sperm. The study provides a reference for the improvement of Qinling giant panda semen cryopreservation.

\section{Material and Methods \\ Semen collection}

Semen from four sexually mature male giant pandas (aged 12-17 years) housed in Shaanxi Wild Animal Research Center (SWARC; Louguantai, Zhouzhi County, Xian city, $34^{\circ} 06 \mathrm{~N}, 108^{\circ} 32 \mathrm{E}$ ) were collected via electrostimulation during the breeding season (from March to May in spring). The giant pandas were anesthetized by injection with ketamine hydrochloride $(8 \mathrm{mg} / \mathrm{kg}, \mathrm{IM})$ and halothane after $12 \mathrm{~h}$ of fasting, and the manually protruding penis was cleaned with pre-warmed sterile saline. The feces was then cleaned from the rectum using enema. To collect semen, the giant pandas were then subjected to 2-3 times electroejaculations (10-90 mA, $5 \mathrm{~s}$ ) with an interval of $2 \mathrm{~s}$ by a probe treated with carboxymethyl cellulose gel. Approximately $5 \mathrm{~mL}$ of fresh semen was collected into $37{ }^{\circ} \mathrm{C}$ pre-warmed sterile cups from each one. This process was performed in compliance with the Wildlife Protection Law of the People's Republic of China and Regulations of Shaanxi Province on the Protection of Wild Animals and Plants. All protocols were approved by the Committee for the Ethics on Animal Care and Experiments in Northwest A\&F University (2017ZX08008005).

\section{Experimental design}

Fresh semen samples were diluted with an extender (Irvine Scientific 90129) at a $1: 1$ (v:v) ratio in $15 \mathrm{~mL}$ tubes placed in a beaker of water at $37^{\circ} \mathrm{C}$. Subsequently, the tubes were incubated at $4{ }^{\circ} \mathrm{C}$ for $3 \mathrm{~h}$. After that, the original cryoprotectant agent (Irvine Scientific 90128) was used to dilute the semen to a final concentration of $400 \times 10^{6}$ spermatozoa/mL, with a final glycerin concentration of $3.33 \%$. Diluted semen was equilibrated at
$4{ }^{\circ} \mathrm{C}$ for $1 \mathrm{~h}$ and aliquoted into eight sets of pre-cooled tubes. Prior to cryopreservation, three sets were supplemented with $50 \mu \mathrm{M}$ RSV, $100 \mu \mathrm{M}$ RSV, or $150 \mu \mathrm{M}$ RSV. Three sets were supplemented with LBP (2 and 4 $\mathrm{mg} / \mathrm{mL})$ and LJP $(1 \mathrm{mg} / \mathrm{mL})$, respectively. and another set was supplemented with a combination of antioxidants, including LJP $(1 \mathrm{mg} / \mathrm{mL})$, RSV $(100 \mu \mathrm{M})$, and LBP $(2 \mathrm{mg} / \mathrm{mL})$. In addition, a set without any antioxidants was set as the control. Finally, diluted semen aliquots were packed into pre-labeled and pre-cooled 250 $\mu \mathrm{L}$ straws, frozen in liquid nitrogen vapor for $2 \mathrm{~min}$ (at $7.5 \mathrm{~cm}$ above liquid nitrogen for $1 \mathrm{~min}, 2.5 \mathrm{~cm}$ above liquid nitrogen for $1 \mathrm{~min}$ ), and stored in nitrogen for longterm preservation [25]. For thawing, frozen straws were removed from liquid nitrogen and immediately placed in a water bath at $37^{\circ} \mathrm{C}$. After shaking for $60 \mathrm{~s}$, the thawed semen was transferred into a $1.5 \mathrm{~mL}$ tube for subsequent experiments.

\section{Semen evaluation Sperm motility test}

After thawing, semen of giant panda was incubated at $37^{\circ} \mathrm{C}$, immediately. Prior to evaluation, the thawed semen was placed in the pre-warmed makler counting chamber (Sefi-Medical, Haifa, Israel) at $37^{\circ} \mathrm{C}$. Subsequently, the total and progressive motility of spermatozoa were examined using an automatic analyzer (Naturegene Sperm Tracker, ANIMAL HST, NatureGene Corp. USA) following the manufacturer's recommendations. Finally, the total and progressive motility of 200 spermatozoa from 5 randomized microscopic fields were evaluated.

\section{Sperm plasma membrane integrity test}

Following the findings of prior studies, the integrity of the sperm plasma membrane was assessed using HOST test [44]. Briefly, hypotonic solutions (25 mM sodium citrate, $75 \mathrm{mM}$ fructose, sterile water) were mixed with rapidly thawed semen of giant pandas, and the mixture was incubated at $37^{\circ} \mathrm{C}$ for $30 \mathrm{~min}$. A total of 200 spermatozoa were counted under an inverted microscope (Soi, Nikon, JPN) to identify the percentage of sperm with swelling tails.

\section{Acrosome integrity assessment}

Acrosome integrity was assessed by fluorescein isothiocyanate-labeled peanut agglutinin (FITC-PNA) staining (Vector Laboratories, USA) [45]. Thawed semen was prepared for sperm smears to a final concentration of 1 $\times 10^{5} / \mathrm{mL}$ and kept dry. The cells were then fixed with methanol for $10 \mathrm{~min}$ and incubated with $30 \mu \mathrm{L}$ FITCPNA $(100 \mu \mathrm{g} / \mathrm{mL})$ at $37{ }^{\circ} \mathrm{C}$ for $30 \mathrm{~min}$ in an environment of light avoidance. Sperm smears were then sealed with a colorless nail polish after washed with PBS in triplicate. 
Finally, more than 200 sperm cells were evaluated using fluorescence microscopy (Axio Observer 3, ZEISS, GER).

\section{Mitochondrial activity analysis}

Tubes containing $1 \mu \mathrm{L}$ PI $(0.02 \mathrm{mg} / \mathrm{mL}$ in PBS solution) (Solarbio, Beijing, China) and $1 \mu \mathrm{L}$ Rhodamine Rh123 $(0.2 \mathrm{mg} / \mathrm{mL}$ in DMSO) (Solarbio, Beijing, China) were stored in dark at room temperature for $10 \mathrm{~min}$ and carefully supplemented with $50 \mu \mathrm{L}$ suspension of sperm that was freeze-thawed for $30 \mathrm{~min}$ [46]. Sperm mitochondrial activity was estimated using an inverted microscope (Soi, Nikon, JPN) at $400 \times$ magnification after sperm smears were prepared with $10 \mu \mathrm{L}$ of the incubated sample. PI negative and Rh123 positive sperm were identified as live sperm with high mitochondrial membrane potential $(\Delta \Psi \mathrm{m})$.

\section{Evaluation of DNA integrity}

DNA integrity was assessed by acridine orange (AO) staining (Solarbio, Beijing, China) [47]. The sperm were washed three times with PBS and then suspended in PBS at a concentration of $2 \times 10^{4} / \mathrm{mL}$. The sperm suspension was placed on clean glass slides and air-dried for the preparation of smears. The smears were then rinsed in a fixative solution (3:1, absolute ethanol: glacial acetic acid) for $5 \mathrm{~min}$. After fixation, the slides were stained with AO solution for $10 \mathrm{~min}$ and thoroughly washed with distilled water. Finally, the air-dried smears were sealed with paraffin oil and analyzed using fluorescence microscopy (Axio Observer 3, ZEISS, GER). At least 300 sperm were counted per smear.

\section{ELISA estimation of ROS, MDA, SOD, GPX, HAase and ACE levels}

The reagent kits were equilibrated at room temperature for $30 \mathrm{~min}$. ROS, MDA, SOD, GPX, HAase and ACE levels were determined by ELISA (Jining, Shanghai, China) in triplicate following the manufacturer's instructions. The optical density of each sample was measured using a multifunctional microplate reader (SPARK, TECAN, CH) at a wavelength of $450 \mathrm{~nm}$. Subsequently, the levels of ROS, MDA, SOD, GSH, HAase and ACE were calculated against each standard curve.

\section{Statistical analysis}

All experiments were replicated at least three times for each group. Data were expressed as means \pm SEM. The data were analyzed with one-way ANOVA followed by LSD using the SPSS Software and the homogeneity of variance was analyzed by Bartlett's test. Statistical significance was set at $P<0.05$.

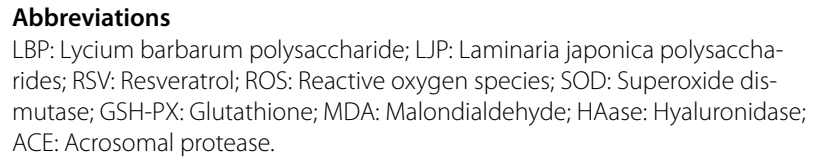
rides; RSV: Resveratrol; ROS: Reactive oxygen species; SOD: Superoxide dismutase; GSH-PX: Glutathione; MDA: Malondialdehyde; HAase: Hyaluronidase; ACE: Acrosomal protease.

\section{Acknowledgements}

We also thank all the technicists for the giant pandas at Louguantai (Shaanxi, China) for their assistance.

\section{Authors' contributions \\ The experiment was designed by LPF and JYP; the samples were collected and performed by ZRX, DHM, ZPP, QH, SCM, MYJ, GCX, ZDH, and SJN; all the data were analyzed by LPF, ZRX, and DHM; All the figures were prepared by LPF. The manuscript was drafted by ZRX; LPF, LYH and JYP revised the manu- script. Finally, all authors reviewed the final manuscript. The authors read and approved the final manuscript.}

\section{Funding}

This research was supported by the Key Research and Development Program of Shaanxi Province (No. 2018ZDXM-NY-036; 2019NY-086), Forestry Scientific Research Program of Shaanxi Province (No. SHLY-2018-09) and Breeding Research Team of Qinling Rare Wild Animals (No. SXLk2020-0303).

\section{Availability of data and materials}

The datasets supporting the conclusions of this study are available from the corresponding author.

\section{Declarations}

Ethics approval and consent to participate

The animal study was reviewed and approved by the Wildlife Protection Law of the People's Republic of China and Regulations of Shaanxi Province on the Protection of Wild Animals and Plants. All protocols were approved by the Committee for the Ethics on Animal Care and Experiments in Northwest A\&F University (Yangling, China). Permission to collect semen sample was obtained from technicists in Research Center for The Qinling Giant Panda (Louguantai, Shaanxi, China ) by verbal. All procedures were handled strictly in accordance with the good animal practice to minimize animal sufferings during semen sampling process.

\section{Consent for publication}

Not applicable.

\section{Competing interests}

All authors declared no competing conflict of interest.

\section{Author details}

${ }^{1}$ College of Veterinary Medicine, Northwest A \& F University, Yangling 712100, Shaanxi, China. ${ }^{2}$ Key Laboratory of Animal Biotechnology, Ministry of Agriculture and Rural Affairs, Northwest A\&F University, Yangling 712100, Shaanxi, China. ${ }^{3}$ Research Center for The Qinling Giant Panda, Rescue Base of Rare Wild Animals in Shaanxi Province, Louguantai 710402, China.

Received: 6 July 2021 Accepted: 20 December 2021

Published online: 07 January 2022

\section{References}

1. Wei F, Fan H, Hu Y. Ailuropoda melanoleuca (Giant Panda). Trends Genet. 2020;36(1):68-9.

2. Zhu L, Hu Y, Qi D, Wu H, Zhan X, Zhang Z, et al. Genetic consequences of historical anthropogenic and ecological events on giant pandas. Ecology. 2013;94(10):2346-57. 
3. Huang Y, Li DS, Zhou YM, Zhou Q, Li RG, Wang CD, et al. Factors Affecting the Outcome of Artificial Insemination Using Cryopreserved Spermatozoa in the Giant Panda (Ailuropoda melanoleuca). Zoo Biol. 2012;31(5):561-73.

4. Comizzoli P. Birth of a Giant Panda Cub After Artificial Insemination with Frozen-Thawed Semen: A Powerful Reminder About the Key Role of Biopreservation and Biobanking for Wildlife Conservation. Biopreserv Biobank. 2020;18(5):349-50.

5. Treulen F, Aguila L, Arias ME, Jofre I, Felmer R. Impact of post-thaw supplementation of semen extender with antioxidants on the quality and function variables of stallion spermatozoa. Anim Reprod Sci. 2019;201:71-83.

6. Peris-Frau P, Soler AJ, Iniesta-Cuerda M, Martin-Maestro A, SanchezAjofrin I, Medina-Chavez DA, Fernandez-Santos MR, Garcia-Alvarez O, Maroto-Morales A, Montoro V et al: Sperm Cryodamage in Ruminants: Understanding the Molecular Changes Induced by the Cryopreservation Process to Optimize Sperm Quality. Int J Molecular Sci. 2020;21 (8):2781.

7. Lv C, Larbi A, Wu G, Hong Q, Quan G. Improving the quality of cryopreserved goat semen with a commercial bull extender supplemented with resveratrol. Animal Reprod Sci. 2019;208:106127.

8. Nouri H, Shojaeian K, Samadian F, Lee S, Kohram H, Lee JI. Using Resveratrol and Epigallocatechin-3-Gallate to Improve Cryopreservation of Stallion Spermatozoa With Low Quality. J Equine Vet Sci. 2018;70:18-25.

9. Sanchez-Rubio F, Fernandez-Santos MR, Castro-Vazquez L, Garcia-Alvarez O, Maroto-Morales A, Soler AJ, et al. Cinnamtannin B-1, a novel antioxidant for sperm in red deer. Animal Reprod Sci. 2018;195:44-52.

10. Reddy VS, Yadav B, Yadav CL, Anand M, Swain DK, Kumar D, et al. Effect of sericin supplementation on heat shock protein 70 (HSP70) expression, redox status and post thaw semen quality in goat. Cryobiology. 2018;84:33-9.

11. Andersen AH, Thinnesen M, Failing K, Goericke-Pesch S. Effect of reduced glutathione (GSH) supplementation to Tris-egg yolk extender on chilled semen variables of dogs. Animal Reprod Sci. 2018;198:145-53.

12. Yang $L$, Gao Z, Lei L, Lv Q, Zhao Q, Li L, et al. Lycium barbarum polysaccharide enhances development of previously-cryopreserved murine two-cell embryos via restoration of mitochondrial function and down-regulated generation of reactive oxygen species. J Reprod Dev. 2019;65(2):163-70

13. Zhu Z, Li R, Fan X, Lv Y, Zheng Y, Hoque SAM, et al. Resveratrol Improves Boar Sperm Quality via 5'AMP-Activated Protein Kinase Activation during Cryopreservation. Oxid Med Cell Longev. 2019;2019:5921503.

14. Yao X, Jiang H, Liang S, Shen X, Gao Q, Xu YN, et al. Laminarin enhances the quality of aged pig oocytes by reducing oxidative stress. J Reprod Dev. 2018:64(6):489-94.

15. Wu PS, Li YS, Kuo YC, Tsai SJ, Lin CC. Preparation and Evaluation of Novel Transfersomes Combined with the Natural Antioxidant Resveratrol. Molecules. 2019;24(3):600.

16. Shi GJ, Zheng J, Wu J, Qiao HQ, Chang Q, Niu Y, et al. Protective effects of Lycium barbarum polysaccharide on male sexual dysfunction and fertility impairments by activating hypothalamic pituitary gonadal axis in streptozotocin-induced type-1 diabetic male mice. Endoc J. 2017:64(9):907-22.

17. Juan ME, Gonzalez-Pons E, Munuera T, Ballester J, Rodriguez-Gil JE, Planas JM. trans-Resveratrol, a natural antioxidant from grapes, increases sperm output in healthy rats. J Nutri. 2005;135(4):757-60.

18. Revel A, Raanani H, Younglai E, Xu J, Han R, Savouret JF, et al. Resveratrol, a natural aryl hydrocarbon receptor antagonist, protects sperm from DNA damage and apoptosis caused by benzo(a)pyrene. Reprod Toxicol. 2001;15(5):479-86.

19. Luo Q, Li Z, Huang X, Yan J, Zhang S, Cai YZ. Lycium barbarum polysaccharides: Protective effects against heat-induced damage of rat testes and $\mathrm{H} 2 \mathrm{O} 2$-induced DNA damage in mouse testicular cells and beneficial effect on sexual behavior and reproductive function of hemicastrated rats. Life sciences. 2006;79(7):613-21.

20. $H u$ JH, Li QW, Li G, Jiang ZL, Bu SH, Yang H, Wang LQ. The cryoprotective effect of trehalose supplementation on boar spermatozoa quality. Anim Reprod Sci. 2009;1 12(1-2):107-18.

21. Cai ZG, An JH, Liu YL, Yie SM, Zhang Y, Li FP, et al. Single layer centrifugation improves the quality of frozen-thawed sperm of giant panda (Ailuropoda melanoleuca). Animal Reprod Sci. 2018;195:58-64.

22. Wang DH, Liu YL, Cai ZG, An JH, Lan JC, Chen JS, et al. Effects of extender type on the quality of post-thaw giant panda (Ailuropoda melanoleuca) semen. Cryobiology. 2020;94:95-9.
23. Ran MX, Li Y, Zhang Y, Liang K, Ren YN, Zhang M, et al. Transcriptome Sequencing Reveals the Differentially Expressed IncRNAs and mRNAs Involved in Cryoinjuries in Frozen-Thawed Giant Panda (Ailuropoda melanoleuca) Sperm. Int J Molecular Sci. 2018;19(10):3066.

24. Ran MX, Zhou YM, Liang K, Wang WC, Zhang Y, Zhang M, et al. Comparative Analysis of MicroRNA and mRNA Profiles of Sperm with Different Freeze Tolerance Capacities in Boar (Sus scrofa) and Giant Panda (Ailuropoda melanoleuca). Biomolecules. 2019;9(9):432.

25. Santiago-Moreno J, Esteso MC, Pradiee J, Castano C, Toledano-Diaz A, O'Brien E, et al. Giant panda (Ailuropoda melanoleuca) sperm morphometry and function after repeated freezing and thawing. Andrologia. 2016:48(4):470-4.

26. Longobardi V, Zullo G, Salzano A, De Canditiis C, Cammarano A, De Luise $L$, et al. Resveratrol prevents capacitation-like changes and improves in vitro fertilizing capability of buffalo frozen-thawed sperm. Theriogenology. 2017:88:1-8.

27. Ren F, Fang Q, Feng T, Li Y, Wang Y, Zhu H, et al. Lycium barbarum and Laminaria japonica polysaccharides improve Cashmere goat sperm quality and fertility rate after cryopreservation. Theriogenology. 2019:129:29-36.

28. Rodriguez-Martinez $\mathrm{H}$, Larsson B, Pertoft $\mathrm{H}$. Evaluation of sperm damage and techniques for sperm clean-up. Reprod Fertil Dev. 1997;9(3):297-308.

29. Zakosek Pipan M, Zrimšek P, Jakovac Strajn B, Pavšič Vrtač K, Knific T, Mrkun J. Macro- and microelements in serum and seminal plasma as biomarkers for bull sperm cryotolerance. Acta Vet Scand. 2021;63(1):25.

30. Buranaamnuay K, Grossfeld R, Struckmann C, Rath D. Influence of cryoprotectants glycerol and amides, combined with antioxidants on quality of frozen-thawed boar sperm. Anim Reprod Sci. 2011;127(1-2):56-61.

31. Flores-Gil VN, Millan De La Blanca MG, Velázquez R, Toledano-Díaz A, Santiago-Moreno J, López-Sebastián A. Influence of testosterone administration at the end of the breeding season on sperm cryoresistance in rams (Ovis aries) and bucks (Capra hircus). Domest Anim Endocrinol. 2020;72:106425.

32. Ahmed H, Jahan $\mathrm{S}$, Ullah H, Ullah F, Salman MM. The addition of resveratrol in tris citric acid extender ameliorates post-thaw quality parameters, antioxidant enzymes levels, and fertilizing capability of buffalo (Bubalus bubalis) bull spermatozoa. Theriogenology. 2020;152:106-13.

33. Macente BI, Apparicio M, Mansano CFM, Tavares MR, Fonseca-Alves CE, Sousa BP, et al. Effect of cryopreservation on sperm DNA fragmentation and apoptosis rates in the testicular tissue of domestic cats. Animal Reprod Sci. 2019;211:106224.

34. Lusignan MF, Li X, Herrero B, Delbes G, Chan PTK. Effects of different cryopreservation methods on DNA integrity and sperm chromatin quality in men. Andrology. 2018;6(6):829-35.

35. Gurler H, Malama E, Heppelmann M, Calisici O, Leiding C, Kastelic JP, et al. Effects of cryopreservation on sperm viability, synthesis of reactive oxygen species, and DNA damage of bovine sperm. Theriogenology. 2016;86(2):562-71.

36. Amaral A, Lourenco B, Marques M, Ramalho-Santos J. Mitochondria functionality and sperm quality. Reproduction. 2013;146(5):R163-74.

37. Medeiros CM, Forell F, Oliveira AT, Rodrigues JL. Current status of sperm cryopreservation: why isn't it better? Theriogenology. 2002;57(1):327-44.

38. Yan B, Zhang $X$, Wang J, Jia S, Zhou Y, Tian J, et al. Inhibitory effect of Lycium barbarum polysaccharide on sperm damage during cryopreservation. Exp Ther Med. 2020;20(4):3051-63.

39. He WH, Zhai XH, Duan XJ, Di HS. Effect of resveratrol treatment on apoptosis and apoptotic pathways during boar semen freezing. J Zhejiang Univ Sci B. 2020;21(6):485-94.

40. Sun L, Fan X, Zeng Y, Wang L, Zhu Z, Li R, Tian X, Wang Y, Lin Y, Wu et al: Resveratrol protects boar sperm in vitro via its antioxidant capacity. Zygote 2020:1-8.

41. Ourique GM, Finamor IA, Saccol EM, Riffel AP, Pes TS, Gutierrez K, et al. Resveratrol improves sperm motility, prevents lipid peroxidation and enhances antioxidant defences in the testes of hyperthyroid rats. Reprod Toxicol. 2013;37:31-9.

42. Zhang G, Yang W, Zou P, Jiang F, Zeng Y, Chen Q, et al. Mitochondrial functionality modifies human sperm acrosin activity, acrosome reaction capability and chromatin integrity. Human reproduction. 2019;34(1):3-11.

43. Yoon S, Chang KT, Cho H, Moon J, Kim JS, Min SH, et al. Characterization of pig sperm hyaluronidase and improvement of the digestibility of 
cumulus cell mass by recombinant PSPAM1 hyaluronidase in an in vitro fertilization assay. Animal Reprod Sci. 2014;150(3-4):107-14.

44. Bloch A, Rogers EJ, Nicolas C, Martin-Denavit T, Monteiro M, Thomas D, et al. Detailed cell-level analysis of sperm nuclear quality among the different hypo-osmotic swelling test (HOST) classes. J Assist Reprod Genet. 2021;38(9):2491-9.

45. Zhu ZD, Fan XT, Lv YH, Zhang N, Fan CN, Zhang PF, et al. Vitamin E Analogue Improves Rabbit Sperm Quality during the Process of Cryopreservation through Its Antioxidative Action. PLoS One. 2015;10(12):e0145383.

46. Mehdipour M, Daghigh Kia H, Nazari M, Najafi A. Effect of lecithin nanoliposome or soybean lecithin supplemented by pomegranate extract on post-thaw flow cytometric, microscopic and oxidative parameters in ram semen. Cryobiology. 2017;78:34-40.

47. Karakus FN, Bulgurcuoglu Kuran S, Solakoglu S. Effect of curcumin on sperm parameters after the cryopreservation. Eur J Obstet Gynecol Reprod Biol. 2021;267:161-6.

\section{Publisher's Note}

Springer Nature remains neutral with regard to jurisdictional claims in published maps and institutional affiliations.

- fast, convenient online submission

- thorough peer review by experienced researchers in your field

- rapid publication on acceptance

- support for research data, including large and complex data types

- gold Open Access which fosters wider collaboration and increased citations

- maximum visibility for your research: over $100 \mathrm{M}$ website views per year

At BMC, research is always in progress.

Learn more biomedcentral.com/submissions 